\title{
1 Pharmacokinetic and safety study of co-administration of albendazole, diethylcarbamazine, Ivermectin and azithromycin for the integrated treatment of Neglected Tropical Diseases
}

Lucy N. John ${ }^{1,2,3}$, Catherine Bjerum ${ }^{4}$, Pere Millat Martinez ${ }^{5}$, Rhoda Likia ${ }^{5}$, Linda Silus ${ }^{5}$, Chilaka Wali $^{2}$, Arthur Elizah ${ }^{5}$, Yashpal S. Chhonker ${ }^{6}$, Veenu Bala ${ }^{6}$, Christopher L. King ${ }^{4}$, Daryl J. Murry ${ }^{6,7}$, Oriol Mitja ${ }^{2,3}$ Michael Marks ${ }^{8,9}$

${ }^{1}$ National Department of Health, Port Moresby, Papua New Guinea,

${ }^{2}$ Barcelona Institute for Global Health - University of Barcelona, Barcelona, Spain,

${ }^{3}$ School of Medicine and Health Sciences, University of Papua New Guinea, Port Moresby, Papua New Guinea

${ }^{4}$ Case Western Reserve University School of Medicine and Veterans Affairs Medical Center, Cleveland, OH, United States,

${ }^{5}$ Lihir Malaria Elimination Program, Newcrest Lihir, New Ireland Province, Papua New Guinea

${ }^{6}$ Clinical Pharmacology Laboratory, Dept. of Pharmacy Practice and Science, Nebraska University Medical Center, Omaha, NE, United States,

${ }^{7}$ Fred and Pamela Buffett Cancer Center, University of Nebraska Medical Center, Omaha, NE, USA,

${ }^{8}$ London School of Hygiene and Tropical Medicine, London, United Kingdom,

${ }^{9}$ Hospital for Tropical Diseases, London, United Kingdom

\section{Brief Summary:}

Pharmacokinetic data are needed to support co-administration of drugs used in the elimination of neglected tropical diseases. We demonstrate that ivermectin, diethylcarbamazine, albendazole and azithromycin can be co-administered without significant drug-drug interactions. This data will facilitate large scale coadministration studies.

\section{ABSTRACT}

Background. Pharmacokinetic data are a pre-requisite to integrated implementation of large-scale mass drug administration (MDA) for neglected tropical diseases (NTDs). We investigated the safety and drug interactions of a combination of azithromycin (AZI) targeting yaws and trachoma, with the newly approved ivermectin, albendazole, diethylcarbamazine (IDA) regime for Lymphatic Filariasis.

Methodology. An open-label, randomized, 3-arm pharmacokinetic interaction study in adult volunteers was carried out in Lihir Island, Papua New Guinea. Healthy adult participants were recruited and randomized to (I) IDA alone, (II) IDA combined with AZI, (III) AZI alone. The primary outcome was lack of a clinically relevant drug interaction. The secondary outcome was the overall difference in the proportion of AEs between treatment arms.

Results. Thirty-seven participants, eighteen men and nineteen women, were randomized and completed the study. There were no significant drug-drug interactions between the study arms. The GMR of $\mathrm{C}_{\max }$, $A U C_{0-t}$, and $A C_{0-\infty}$ for IVM, DEC, ALB-SOX, and AZI were within the range of 80-125\% (GMR for AUC $0-$ $\infty$ for IVM, 87.9; DEC, 92.9; ALB-SOX, 100.0; and AZI, 100.1). There was no significant difference in the frequency of AEs across study arms (AZI and IDA alone arms 9/12 (75\%), co-administration arm 12/13 $(92 \%) ; p=0.44)$. All AEs were grade 1 and self-limiting.

Conclusions. Co-administration of AZI with IDA did not show evidence of significant drug-interactions. There were no serious AEs in any of the study arms. Our data support further evaluation of the safety of integrated MDA for NTDs. 
50 Clinical Trials Registration. NCT03664063

51 Keywords: yaws, lymphatic filariasis, mass drug administration, co-administration

\section{Corresponding author:}

53 L. N. John National Department of Health P.O.BOX 807, Waigani NCD lucyninmongojohn@gmail.com or

54 lucy_john@health.gov.pg

\section{Alternative Corresponding Author:}

56 M. Marks London School of Tropical Medicine and Hygiene, Clinical Research Department, London

57 School of Hygiene \& Tropical Medicine, Keppel Street, London, WC1E 7HT, michael.marks@lshtm.ac.uk

58 Word count (2,680 words)

59 Abstract 250 words

60 Number of figures (2), tables (3), supplementary figures (1), supplementary tables (1) 


\section{BACKGROUND}

62 Mass drug administration (MDA) is the mainstay of control programs for many neglected tropical diseases

63 (NTDs) including lymphatic filariasis (LF), soil-transmitted helminths (STH), trachoma and yaws [1-3]. In

64 many countries, including Papua New Guinea (PNG), most NTD control programs run separately and

65 deliver separate MDA campaigns for each targeted disease. However, conducting separate MDA

66 campaigns for each NTD involves added complexity and increases economic and logistic costs. Studies in

67 other settings have explored combining MDA for LF and schistosomiasis, which appears to be safe and

68 allows programs to achieve considerable cost-savings [4]. Expanding opportunities for integration of MDA

69 campaigns is therefore an attractive strategy for Ministries of Health and partner organizations for both

70 logistical and economic reasons.

72 Lymphatic filariasis is an endemic nematode infection, most commonly caused by Wuchereria bancrofti,

73 and affects 120 million people worldwide. For the last 20 years, the main LF elimination strategy has

74 consisted of repeated rounds of MDA with albendazole (ALB) and either diethylcarbamazine (DEC) or

75 ivermectin (IVM). However, recent studies have shown that single-dose combination therapy with all three

76 drugs, IVM, DEC, ALB (IDA) is superior to the previous two-drug combinations, and may help accelerate

77 LF elimination [1]. In light of this emerging data on both, safety and efficacy, WHO has provided alternative

78 guidelines recommending IDA based MDA in countries endemic for LF outside sub-Saharan Africa [5].

80 Trachoma is caused by Chlamydia trachomatis infection and is the leading infectious cause of blindness

81 worldwide. The macrolide antibiotic azithromycin (AZI) has been demonstrated to be highly safe and

82 effective as MDA for trachoma [6], and now forms a cornerstone of the WHO SAFE strategy [2]. Recently

83 single doze AZI has also been shown to be effective against yaws and is now recommended by WHO for

84 this indication[3,7].

85

Pharmacokinetic (PK) data are needed to ensure that there are no significant drug-drug interactions that might impact either the safety or efficacy of co-administration of the new IDA regimen and AZI. PK data

88 formed an important part of the safety data collected prior to large scale field studies of the IDA regimen

89 and have shown no clinically important effect on any of the drug levels [8,9]. Previous PK studies examining

90 the interaction between IVM, ALB and AZI have not identified clinically meaningful drug-drug interactions

91 and small-scale field implementation studies have suggested co-administration is safe [10-12]. There is no

92 PK data on co-administration of DEC and AZI with or without the addition of IVM or ALB. We therefore

93 conducted a PK study amongst healthy volunteers to assess the safety and drug interactions of co-

94 administration of AZI alongside IDA in PNG.

95

96

97 
METHODS

\section{Study setting and participants}

100 We undertook an open-label, parallel-group, randomized study with 3 treatment arms at the Lihir Medical

101 Centre between Sept 15 and Oct 15, 2018. Participants were recruited from Kunaye 1, Kunaye 2, Putput

102 and Zuen villages of Lihir Island, New Ireland Province, PNG. All individuals provided written informed 103 consent to participate in the study. The Medical Research Advisory committee of PNG (MRAC 17.19) and

104 the institutional Review Board of Case Western University approved the study. The trial was registered at 105 ClinicalTrials.gov (NCT03664063).

Eligible participants were adult healthy volunteers aged 18-70 years who reported no significant past medical history and no current acute illnesses. At enrollment participants underwent a standardized medical

109 examination and blood and urine tests. Exclusion criteria were alanine transaminase (ALT), aspartate 110 transaminase (AST), or creatinine $>1.5$ times the upper limit of normal; hemoglobin levels $<7 \mathrm{gm} / \mathrm{dL}$; 111 abnormal $(>++)$ urine leucocytosis or glucosuria and pregnancy.

\section{Randomization and masking}

114 Eligible participants were randomly assigned by use of a computer generated randomization sequence 115 stratified by sex to receive one of three treatment regimens: (ARM-I) IVM $200 \mu \mathrm{g} / \mathrm{kg}+$ DEC $6 \mathrm{mg} / \mathrm{kg}+$ ALB $116400 \mathrm{mg}$, or (ARM-II) IVM $200 \mu \mathrm{g} / \mathrm{kg}+$ DEC $6 \mathrm{mg} / \mathrm{kg}+$ ALB $400 \mathrm{mg}+$ AZI $30 \mathrm{mg} / \mathrm{Kg}$, or (ARM-III) AZI 30 $117 \mathrm{mg} / \mathrm{Kg}$. Randomization was done in permuted blocks of six and in a 1:1:1 ratio. The allocation was 118 concealed from investigators by use of opaque, sealed and sequentially numbered envelopes that were 119 opened after the study team decided to enroll the participant. Laboratory technicians were unaware of 120 participants' treatment allocation. All participants received directly observed treatment, but masking was 121 not possible for logistical reasons.

\section{Procedures}

124 The primary outcome was lack of a clinically relevant pharmacokinetic drug interactions, defined as 125 geometric mean ratios (GMRs) within the conventional acceptance range of 80-125 for the Cmax, $\mathrm{AUC}_{0-\mathrm{t}}$, 126 and $\mathrm{AUCO}_{-\infty}$ between treatment arms. GMR was used as previous studies have shown the 127 pharmacokinetics of IVM, DEC, and ALB to be highly variable (CV greater than 30\%). The secondary 128 outcome was the difference in the overall proportion of AEs between treatment arms.

130 The study team conducted local visits to communities to explain the purpose and the procedures involved 131 in the study and volunteers were provided detailed information. For the purpose of the study, all participants 132 were admitted the day before treatment administration for a period of 72 hours for blood collections and 133 close monitoring of adverse event $(\mathrm{AE})$. At baseline we tested for malaria antigen (CareStart ${ }^{\circledR}$ Malaria 134 PF/PAN rapid diagnostic test, ACCESSBIO), syphilis serology (DPP ${ }^{\circledR}$ syphilis screen \& confirm Assay, 
135 CHEMBIO), W. bancrofti antigen (Alere ${ }^{\circledR}$ Filarial Test Strips, ABBOTT), for liver function fests, kidney 136 function fests, full blood count, and urinalysis (Multistix 10 SG, Bayer/Seimens). Female participants had a 137 pregnancy test performed. Participants were fasted overnight and medication was administered at $0700 \mathrm{~h}$ 138 after breakfast. Blood draws for PK testing were performed at baseline, 1, 2, 3, 4, 6, 8, 12 hours (using 139 intravenous cannulas) and at 24,48 , and 72 hours using venipuncture in keeping with previous similar 140 studies [2,8]. Participants were monitored for AEs on the basis of physical examinations including recording 141 blood pressure (BP), pulse rate, respiratory rate and temperature every 6 hours for the first 24 hours and 142 then every 12 hours up to 72 hours after drug administration. We tested for full blood count, liver and kidney

143 function, and urinalysis daily for the 72 hours. We conducted an additional safety visit in the community at 144 day 7.

146 Blood samples for PK analysis were stored at a temperature of $-15{ }^{\circ} \mathrm{C}$ at site laboratory and were then 147 shipped on dry ice to the University of Nebraska Medical Center. Plasma concentrations of DEC, ALB, ALB148 SOX (Albendazole-Sulphoxide), ALB-SON (Albendazole-sulphone) and IVM were determined using a 149 validated liquid chromatography-mass spectrometric (LC-MS/MS) methods as previously reported[8,9]. AZI 150 plasma concentrations were determined using a validated LC-MS/MS assay (under preparation for 151 publication). The PK parameters of DEC, ALB, ALB-SOX, ALB-SON, IVM and AZI were calculated using 152 non-compartmental analysis (NCA) using Phoenix WinNonlin-8.1 (Certara, Princeton, NJ, USA). The 153 maximum concentration $\left(\mathrm{C}_{\max }\right)$, and time to $\mathrm{C}_{\max }\left(\mathrm{T}_{\max }\right)$ were determined directly from the plasma 154 concentration-time data. The area under the curve ( $\left.\mathrm{AUC}_{0 \text {-inf }}\right)$, was estimated using the trapezoidal method 155 from 0 to tlast and extrapolation from tlast to infinity $\left(\mathrm{AUC}_{0-\infty}\right)$ based on the observed concentration at the last 156 time point divided by the terminal elimination rate constant $\left(\lambda_{z}\right)$. The half-life $\left(t \frac{1}{2}\right)$ was calculated using the 157 formula of $0.693 / \lambda_{z}$. Apparent volume of distribution $(V z / F)$ and clearance $(C L / F)$ for each drug was 158 calculated using standard equations. Values of $\mathrm{C}_{\max }, \mathrm{AUC}_{0-\mathrm{t}}$, and $\mathrm{AUC}_{0_{-\infty}}$ were normalized to $\mathrm{mg} / \mathrm{kg}$ doses 159 of $4 \mathrm{mg} / \mathrm{kg}$ for ALB, $6.0 \mathrm{mg} / \mathrm{kg}$ for DEC (or $3.0 \mathrm{mg} / \mathrm{kg}$ after salt normalization), $200 \mu \mathrm{g} / \mathrm{kg}$ for IVM and 30 $160 \mathrm{mg} / \mathrm{kg}$ for $\mathrm{AZI}$, to reduce variability in PK parameters resulting from the differing $\mathrm{mg} / \mathrm{kg}$ doses administered 161 to each subject.

163 Adverse Events were defined as any one of the following: an increase in ALT, AST, or creatinine $>1.5$ times 164 the upper limit of normal, tympanic temperature $>37.8^{\circ} \mathrm{C}$, or $\mathrm{BP}<90 / 60$. Subjective AEs were assessed by 165 interviews and were defined as any new symptoms and worsening of pre-existing symptoms. Severity was 166 assessed using the GRADE system established in the Common Terminology Criteria for Adverse Events. 167 In all participants reporting a grade $\geq 2 \mathrm{AE}$, a targeted physical examination was conducted by a study 168 clinician. If appropriate, additional diagnostic testing and treatment was provided through the Lihir Medical 169 Centre. Any medical treatment required was provided free of charge to participants. All data was collected 170 using standardized data collection forms. Data was double entered into a REDCap database. 


\section{Pharmacokinetic and Pharmacodynamic Analysis}

173 Power calculations indicated that 42 participants (14 subjects per arm) would give a power of $80 \%$ to test

174 the hypothesis that the primary outcome of a bioequivalence between test groups between $80-120 \%$ of 175 geometric mean ratio (see below) based on previous PK modelling studies [8] and European Medicines

176 Agency guidelines [13] with the assumption that $10 \%$ of participants would be lost to follow-up. For analysis 177 of the primary outcome (lack of clinically relevant pharmacokinetic interactions), we estimated one-sided $17890 \% \mathrm{Cl}$ for the geometric mean ratios (GMRs) of the experimental regimen and the reference regimens.

179 Descriptive comparisons of PK parameters between arms were performed using the Kruskal-Wallis test 180 using the JMP software (Ver. 14.0, Cary, NC, USA) and comparison of GMRs of the main PK parameters 181 and $90 \% \mathrm{Cl}$ were estimated, after log transformation of within-subject using Phoenix WinNonlin-8.1 182 (Certara, Princeton, NJ, USA). The data obtained in this study were compared according to Food and Drug 183 Administration and European Medicines Agency guidelines (EMA) (90\% Cl, 80\%-125\% for $\mathrm{AUC}_{0-\infty}$ and 184 Cmax)[13,14]. According to the EMA guideline, the wider equivalence range could be considered for highly 185 variable drugs (intra-subject coefficient of variation $>30 \%$ ). Previous studies have shown the substantial 186 PK variability with coefficient of variations for $\mathrm{AUC}_{0-\infty}, \mathrm{AUC}_{0-\mathrm{t}}$, and $\mathrm{C}_{\max }$ greater than $30 \%$ for $\mathrm{DEC}$, IVM and 187 AZI and $C_{\max }$ greater than $50 \%$ for ALB and its active metabolite $[12,13]$.

\section{Statistical Analysis}

For analysis of the AEs outcomes, we calculated the frequency of each AE by study arm. We grouped diarrhea, abdominal pain and nausea together into a single $A E$ category. Differences between arms were assessed using a Chi-Square test. All statistical analysis was conducted in $\mathrm{R}$ version 3.4 .2 (The $\mathrm{R}$ Foundation for Statistical Computing) [15].

\section{RESULTS}

\section{Study Enrolment and Flowchart}

200 Forty-two individuals were screened for inclusion into the study. Three participants were excluded (pregnant $201 \mathrm{n}=1$, acute febrile illness $\mathrm{n}=1$, unable to obtain venous access $n=1$; Figure 1). Thirty-nine (39) 202 participants met study inclusion and 37 completed the full study. Two participants were excluded after 203 screening ( $n=1$ in ARM-II, consumed alcohol following treatment; $n=1$ in ARM-III withdrew and did not 204 receive study drugs). The three study arms were well balanced with regards to demographic characteristics. 205 The mean age (years+/-SD) of the 37 participants that completed the study was $29.2(10.6)$ and 19 (51.4\%) 206 were female (Table 1). Overall 10 (27\%) participants had serological evidence of yaws, and $8(21.6 \%)$ had 207 serological evidence of lymphatic filariasis. 
210 PK parameters for IDA alone (ARM-I), IDA+ AZI (ARM-II) or AZI alone (ARM-III) are shown in Table 2. The 211 median elimination $\mathrm{t}_{1 / 2}$ and time to peak concentration was similar for DEC, ALB-SOX, IVM and AZI when 212 given alone or in combination. Median values for any comparison were not different between study arms 213 ( $>0.05)$. Ranges for each PK parameter are shown in Supplementary Table 1. Distribution of dose 214 adjusted $\mathrm{C}_{\max }$ and $\mathrm{AUC}_{0-\mathrm{t}}$ of study drugs by study ARM with individual data points are shown in 215 Supplementary Figure 1.

217 The mean plasma concentration-time profiles of ALB, ALB-SOX (the active metabolite of ALB), ALB-SON, 218 DEC, IVM and AZI are shown in Figure 2. Geometric mean ratios (GMR) of parameters in the experimental 219 arm (IDA + AZI) versus the reference arms (IDA and AZI alone) are presented with $90 \%$ confidence intervals 220 (Cls) in Figure 3. $\mathrm{C}_{\max }, \mathrm{AUC}_{0-\mathrm{t}}$, and $\mathrm{AUC}_{0-\infty}$ for each analyte were dose normalized. The $\mathrm{GMR}$ of $\mathrm{C}_{\max }$, $221 \mathrm{AUC}_{0-\mathrm{t}}$, and $\mathrm{AUC}_{0-\infty}$ for DEC, IVM, ALB-SOX and AZI were within the range of $80-125 \%$, and the $90 \% \mathrm{Cls}$ 222 partly overlap the range of $50-200 \%$ that reflects the inter subject variability. For ALB, which is rapidly 223 metabolized to ALB-SOX, the GMR of $\mathrm{C}_{\max }, \mathrm{AUC}_{0-\mathrm{t}}$, and $\mathrm{AUC}_{0-\infty}$, were within the range of $80-125 \%$ (data 224 not shown).

\section{Adverse Events}

227 Overall, 30 (81.0\%) of 37 participants developed at least 1 AE (Table 3). AEs were reported by 9/12 (75\%) 228 in ARM-I, 12/13 (92\%) in ARM-II, and 9/12 (75\%) in ARM-III, however this difference was not significant $(p=0.44)$. All AEs reported in the study were Grade 1 and self-limiting. No serious AEs occurred in any of the study arms. No participants required treatment for any AE. A total of $372 \mathrm{AE}$ assessments were conducted; the most common AEs were headache (11 episodes, 3.0\%), GI upset ( 13 episodes, 3.5\%), and asymptomatic transient hypotension (15 episodes, 4.0\%) (Table 2). Biochemically, the highest recorded ALT and AST were 85iu/L and 76iu/L respectively at 24 hours post treatment and both resolved by 48 hours. The highest creatinine was 158umol/L at 24 hours which also resolved by 48 hours.

\section{DISCUSSION}

237 Our findings show that co-administration of AZI alongside the new triple-drug IDA regime for LF was tolerable and without any evidence of significant drug-drug interactions. The GMR values of PK parameters for IVM, DEC and ALB or ALB metabolites, were not significantly altered by the co-administration of AZI, and values were similar to those seen in previous studies[8,9,16]. These results suggest that $A Z I$ has no clinically relevant effect on the PK of IVM, DEC and ALB. Moreover, there was no change in the PK for AZI when administered in this combination regimen. There was considerable variability in plasma ALB and IVM drug levels among individuals as has been previously reported[8,9]. Evidence before this study showed

244 that combinations for NTDs were safe in terms of PK interactions between AZI and IVM, IVM and ALB, IDA 245 drugs, and IVM, ALB and AZI[8-11,16]. The added value of this study is that for the first time we report on 246 the safety of a quadruple combination of IDA and AZI. 
248 This study also showed no serious AEs in any of the 3 study arms. Mild AEs (grade 1) were frequent in all 249 arms but self-limiting. Of participants who were treated with combined treatment $92 \%$ reported mild AEs 250 that were mainly gastrointestinal, compared to $75 \%$ of participants who received IDA or AZI alone. Whilst, 251 given the small sample size, we cannot preclude a risk of rarer more serious AEs due to co-administration, 252 our data provides substantial reassurance that co-administration is well tolerated.

254 The main limitation of this study is the study sample size, which was only designed to exclude significant 255 drug-drug interactions. A larger sample size is required to better understand whether the trend toward a 256 higher rate of AEs with co-administration will be borne out and to assess for rarer AEs which may occur. 257 Secondly, we did not assess the impact of co-administration on the efficacy of any of the drugs but given 258 the absence of any significant drug-drug interactions it seems highly unlikely that co-administration would 259 impact efficacy. Thirdly, we did not systematically measure acceptability on the challenge of swallowing a 260 large number of tablets, but we observed that participants' acceptance was very high. Finally, our population 261 was limited to adults. Data in paediatric populations would be of value to further support the case for 262 integrated MDA, however we would not expect any significant interaction in children based on the results 263 in adults, although optimal dosing in children may be more variable in MDA campaigns. It should be noted 264 that IVM is currently not given to children $<15 \mathrm{~kg}$ and/or $<5$ years of age.

Our findings provide strong evidence on the lack of pharmacokinetic drug interactions and tolerability of coadministration of IDA with AZI. This data paves the way for integrated MDA programs targeting LF, STH, trachoma, scabies and yaws $[18,19]$. The benefits of MDA integration include increased coverage and geographic reach of national NTD programs, whilst achieving financial and programmatic savings. Integrated MDAs will be of particular value in countries such as Papua New Guinea where these diseases are co-endemic and where the cost of individual MDA is particularly high compared to other settings [17]. Field studies are now planned to further evaluate the safety of co-administration within a programmatic context.

\section{Notes}

Financial support. Funding for the study was provided by the International Trachoma Initiative.

Potential conflicts of interest. The authors declare no relevant conflict of interest.

Contributions. LJ, MM, CB, CK, OM conceived the study. LJ, CB, OM, MM, PM, RL, LS, CW and AE conducted the study. CK, YC, VB and DM performed the PK analysis. LJ, OM and MM drafted the manuscript. All authors contributed to revising the manuscript.

Acknowledgements. Participants who willingly participated in this study are acknowledged and thanked for their contribution. The Papua New Guinea National Department of Health and the Lihir Medical Centre are hereby thanked for their support in conducting this study. 
288

289 
291 1. King CL, Suamani J, Sanuku N, et al. A Trial of a Triple-Drug Treatment for Lymphatic Filariasis. N $292 \quad$ Engl J Med 2018; 379:1801-1810.

293 2. Emerson PM, Burton MJ, Solomon AW, Bailey R, Mabey DC. The SAFE strategy for trachoma

3. Mitjà $\mathrm{O}$, Houinei $\mathrm{W}$, Moses $\mathrm{P}$, et al. Mass Treatment with Single-Dose Azithromycin for Yaws. $\mathrm{N}$ control: using operational research for policy, and implementation. Bull World Health Organ 2006; 84:613-619.

Engl J Med 2015; 372:703-710.

4. Evans D, Mcfarland D, Adamani W, et al. Cost-effectiveness of triple drug administration (TDA) with praziquantel, ivermectin and albendazole for the prevention of neglected tropical diseases in Nigeria. Ann Trop Med Parasitol 2011; 105:537-547.

5. WHO | Guideline - Alternative mass drug administration regimens to eliminate lymphatic filariasis. Available at: http://www.who.int/lymphatic_filariasis/resources/9789241550161/en/. Accessed 8 December 2019.

6. Solomon AW, Holland MJ, Alexander NDE, et al. Mass Treatment with Single-Dose Azithromycin for Trachoma. N Engl J Med 2004; 351:1962-1971.

7. The World Health Organisation. Eradication of yaws - the Morges Strategy. Wkly Epidemiol Rec 2012; 87:189-194.

8. Thomsen EK, Sanuku N, Baea M, et al. Efficacy, Safety, and Pharmacokinetics of Coadministered Diethylcarbamazine, Albendazole, and Ivermectin for Treatment of Bancroftian Filariasis. Clin Infect Dis 2016; 62:334-341.

9. Bjerum CM, Ouattara AF, Aboulaye M, et al. Efficacy and Safety of a Single Dose of Ivermectin, Diethylcarbamazine, and Albendazole for Treatment of Lymphatic Filariasis in Côte d'Ivoire: An Open-label Randomized Controlled Trial. Clin Infect Dis Available at: https://academic.oup.com/cid/advance-article/doi/10.1093/cid/ciz1050/5602915. Accessed 8 December 2019.

10. El-Tahtawy A, Glue P, Andrews EN, Mardekian J, Amsden GW, Knirsch CA. The Effect of Azithromycin on Ivermectin Pharmacokinetics-A Population Pharmacokinetic Model Analysis. PLOS Negl Trop Dis 2008; 2:e236.

11. Amsden GW, Gregory TB, Michalak CA, Glue P, Knirsch CA. Pharmacokinetics of Azithromycin and the Combination of Ivermectin and Albendazole When Administered Alone and Concurrently in Healthy Volunteers. Am J Trop Med Hyg 2007; 76:1153-1157.

12. Coulibaly YI, Dicko I, Keita M, et al. A cluster randomized study of the safety of integrated treatment of trachoma and lymphatic filariasis in children and adults in Sikasso, Mali. PLoS Negl Trop Dis 2013; 7:e2221.

14. Research C for DE and. Statistical Approaches to Establishing Bioequivalence. 2019. Available at: http://www.fda.gov/regulatory-information/search-fda-guidance-documents/statistical-approachesestablishing-bioequivalence. Accessed 8 December 2019. 
331

332

333

334

335

336

337

338

339

340

341

342

343

344

345

346

347

348

349

350

351

352

353

354

355

356

357

358

359

360

361

362

363

364

365

366

367

368

369

370

371

372

373

374

375

376

377

378

379

380

381

382
15. R Core Team. R: A Language and Environment for Statistical Computing. Vienna, Austria: R Foundation for Statistical Computing, 2017. Available at: https://www.R-project.org/.

16. Awadzi K, Edwards G, Duke BOL, et al. The co-administration of ivermectin and albendazole-safety, pharmacokinetics and efficacy against Onchocerca volvulus. Ann Trop Med Parasitol 2003; 97:165-178.

17. Fitzpatrick C, Fleming FM, Madin-Warburton M, et al. Benchmarking the Cost per Person of Mass Treatment for Selected Neglected Tropical Diseases: An Approach Based on Literature Review and Meta-regression with Web-Based Software Application. PLoS Negl Trop Dis 2016; 10:e0005037. 


\section{Figure Legends}

385

Figure 1: Study enrolment flowchart

386

387 Figure 2: Drug concentration vs time curve plots for subjects on the IDA, IDA+AZI and AZI alone 388 study arms.

389 Overlay of mean ( \pm SD) plasma concentration-time profiles of (a) ALB, (b) ALB -SOX (c) ALB-SON, (d) DEC (e) IVM, and (f) AZI after a single dose separated by study ARM (IDA, $n=12$, IDA+AZI, $n=13, A Z I, n=13$ ).

Figure 3. Forest plots of the geometric mean ratios ( $\pm 90 \%$ confidence intervals $[\mathrm{Cl}])$ of the drug

395 transformed $\mathrm{C}_{\max }$ and $\mathrm{AUC}_{0-\mathrm{t}}$ and $\mathrm{AUC}_{0-\mathrm{t}}$ and $\mathrm{AUC}_{0-\infty .}$

The vertical dashed lines represent the EMA and US FDA criteria of 80 to $125 \%$ for assuming no effect boundary. 


\begin{tabular}{|c|c|c|c|}
\hline & $\begin{array}{l}\text { IDA } \\
\text { Alone (ARM-I, } \\
\mathrm{N}=12)\end{array}$ & $\begin{array}{l}\text { IDA and } \\
\text { Azithromycin } \\
(\text { ARM-II, N=13) }\end{array}$ & $\begin{array}{l}\text { Azithromycin } \\
\text { Alone (ARM-III, } \\
\mathrm{N}=12 \text { ) }\end{array}$ \\
\hline \multicolumn{4}{|l|}{ Age } \\
\hline Mean (SD) & $25.6(11.4)$ & $32.3(11.5)$ & $29.3(8.4)$ \\
\hline Range & $18.0-59.0$ & $20.0-55.0$ & $21.0-52.0$ \\
\hline \multicolumn{4}{|l|}{ Sex } \\
\hline Male & $6(50.0 \%)$ & $6(46.2 \%)$ & $6(50.0 \%)$ \\
\hline Female & $6(50.0 \%)$ & $7(53.8 \%)$ & $6(50.0 \%)$ \\
\hline \multicolumn{4}{|l|}{ Weight } \\
\hline Mean (SD) & $61.2(9.2)$ & $64.3(13.3)$ & $66.3(15.7)$ \\
\hline Range & $46.0-73.0$ & $51.0-92.0$ & $41.0-93.0$ \\
\hline \multicolumn{4}{|l|}{ BMI } \\
\hline Mean (SD) & $22.8(3.3)$ & $24.8(5.1)$ & $25.7(5.8)$ \\
\hline Range & $18.7-29.2$ & $19.1-35.8$ & $17.3-36.3$ \\
\hline \multicolumn{4}{|l|}{ DEC Dose } \\
\hline Mean (SD) & $366.7(57.7)$ & $380.8(72.3)$ & NA \\
\hline \multicolumn{4}{|l|}{ Albendazole Dose } \\
\hline Mean (SD) & 400 & 400 & NA \\
\hline \multicolumn{4}{|l|}{ Ivermectin Dose } \\
\hline Mean (SD) & $12.8(2.3)$ & $13.4(2.3)$ & NA \\
\hline \multicolumn{4}{|l|}{ Azithromycin Dose } \\
\hline Mean (SD) & NA & $1750(204.1)$ & $1770.8(270.9)$ \\
\hline \multicolumn{4}{|l|}{ DPP Result } \\
\hline Negative & $6(50.0 \%)$ & $6(46.2 \%)$ & $9(75.0 \%)$ \\
\hline $\begin{array}{l}\text { Treponema Positive and } \\
\text { Non-Treponema Negative }\end{array}$ & $1(8.3 \%)$ & $2(15.4 \%)$ & $3(25.0 \%)$ \\
\hline $\begin{array}{l}\text { Treponema Positive and } \\
\text { Non-Treponema Positive }\end{array}$ & $5(41.7 \%)$ & $5(38.5 \%)$ & $0(0.0 \%)$ \\
\hline \multicolumn{4}{|l|}{ Filariasis Test Strip Result } \\
\hline Negative & $8(66.7 \%)$ & $11(84.6 \%)$ & $10(83.3 \%)$ \\
\hline Positive & $4(33.3 \%)$ & $2(15.4 \%)$ & $2(16.7 \%)$ \\
\hline
\end{tabular}

403

404

405

406

407

408

409

410

411

412

413

414

415

416

417

418

419 
Table 2: Pharmacokinetic parameters of the study drugs when administered in either a three drug (IDA), a four drug combination (IDA+AZI) or AZI alone.

\begin{tabular}{|c|c|c|c|c|c|c|c|c|}
\hline Parameter & ALB- & sox & $\mathrm{DE}$ & & IV & & A & \\
\hline & IDA & IDA+AZI & IDA & IDA+AZI & IDA & IDA+AZI & AZI & IDA+AZI \\
\hline $\mathrm{C}_{\max }(\mathrm{ng} / \mathrm{mL})$ & 391.6 & 443.6 & 1368.9 & 1539.1 & 96.6 & 83.6 & 1190.6 & 1648.8 \\
\hline $\mathrm{T}_{\max }(\mathrm{hr})$ & 5 & 6 & 3 & 4 & 6 & 6 & 3.5 & 4 \\
\hline Half-life $\left(t_{1 / 2}\right)$ & 7.3 & 8.1 & 10.7 & 9.9 & 24.3 & 33.1 & 32.1 & 29.9 \\
\hline $\mathrm{AUC}_{0-\mathrm{t}}\left(\mathrm{ng}{ }^{*} \mathrm{hr} / \mathrm{mL}\right)$ & 5484 & 5902.2 & 22967.6 & 21227.6 & 1856.1 & 1576.1 & 11332.8 & 14532 \\
\hline $\mathrm{AUC}_{0-\infty}\left(\mathrm{ng}{ }^{*} \mathrm{hr} / \mathrm{mL}\right)$ & 5487.8 & 5921.6 & 23299.3 & 21397.3 & 2178.9 & 2019.9 & 13950.2 & 17298.6 \\
\hline $\mathrm{V}_{\mathrm{z} / \mathrm{F}}(\mathrm{L})$ & 788.5 & 783.4 & 127.2 & 136.6 & 213.9 & 332.6 & 5484.2 & 5504.3 \\
\hline $\mathrm{Cl} / \mathrm{F}(\mathrm{L} / \mathrm{hr})$ & 73 & 68.2 & 7.7 & 9.2 & 5.6 & 6 & 129.5 & 101.2 \\
\hline $\begin{array}{l}\mathrm{C}_{\max } \text { adjusted to dose } \\
\text { (ng/mL) }\end{array}$ & 222.3 & 278.7 & 1420.8 & 1542.9 & 96.7 & 78.9 & 1312.5 & 1905.9 \\
\hline $\begin{array}{l}\text { AUC }_{0-\mathrm{t}} \text { adjusted to } \\
\text { Dose }(\mathrm{ng} \mathrm{hr} / \mathrm{mL})\end{array}$ & 3103.7 & 4712.8 & 22750.8 & 23147.9 & 1746 & 1567.2 & 12511 & 14778 \\
\hline $\begin{array}{l}\text { AUC }_{0-\infty} \text { adjusted to } \\
\text { dose }\left(\mathrm{ng}^{*} \mathrm{hr} / \mathrm{mL}\right)\end{array}$ & 3151.3 & 4731.3 & 23079.6 & 23333.6 & 2047.5 & 1962.8 & 16706.8 & 17208 \\
\hline
\end{tabular}

423 Data presented are the median values for each pharmacokinetic parameter.

424 Data are median. $\mathrm{T}_{1 / 2}$ terminal half-life, $\mathrm{T}_{\max }$ time of maximum plasma concentration, $\mathrm{C}_{\max }$ maximum plasma concentration, $\mathrm{AUC}$ area 425 under the concentration-time curve, Vz/F apparent volume of distribution, CL/F apparent clearance.

426 ALB-SOX, albendazole sulfoxide, DEC, diethylcarbamazine, IVM, ivermectin, AZI, azithromycin.

427 IDA, three drug combination (DEC 6mg/kg + IVM 200ug/kg + ALB 400mg); IDA+AZI, four drug combination (IVM 200 $\mu \mathrm{gg} / \mathrm{kg}+\mathrm{DEC}$ $4286 \mathrm{mg} / \mathrm{kg}+$ ALB $400 \mathrm{mg}+\mathrm{AZI} 30 \mathrm{mg} / \mathrm{kg}$ ); or AZI (AZI alone $30 \mathrm{mg} / \mathrm{kg}$ ). 
Table 3: Adverse events experienced in each of the three study arms (IDA, IDA+AZI, AZI alone).

\begin{tabular}{|c|c|c|c|c|c|}
\hline & $\begin{array}{l}\text { IDA Alone } \\
\text { (ARM-I, } \\
\mathrm{N}=12 \text { ) }\end{array}$ & $\begin{array}{l}\text { IDA+AZI (ARM- } \\
I I, N=13)\end{array}$ & $\begin{array}{l}\text { AZI Alone } \\
\text { (ARM-III, } \\
\mathrm{N}=12)\end{array}$ & $\begin{array}{l}\text { Total } \\
(\mathrm{N}=37)\end{array}$ & $p$ value \\
\hline Fever & $0(0.0 \%)$ & $1(7.7 \%)$ & $0(0.0 \%)$ & $1(2.7 \%)$ & 0.39 \\
\hline Headache & $3(25.0 \%)$ & $2(15.4 \%)$ & $3(25.0 \%)$ & $8(21.6 \%)$ & 0.80 \\
\hline GI Upset & $2(16.7 \%)$ & $5(38.5 \%)$ & $2(16.7 \%)$ & $9(24.3 \%)$ & 0.34 \\
\hline Myalgia & $0(0.0 \%)$ & $2(15.4 \%)$ & $0(0.0 \%)$ & $2(5.4 \%)$ & 0.14 \\
\hline Itch & $1(8.3 \%)$ & $0(0.0 \%)$ & $0(0.0 \%)$ & $1(2.7 \%)$ & 0.34 \\
\hline Cough & $0(0.0 \%)$ & $1(7.7 \%)$ & $0(0.0 \%)$ & $1(2.7 \%)$ & 0.39 \\
\hline Hypotension* & $5(41.7 \%)$ & $1(7.7 \%)$ & $3(25.0 \%)$ & $9(24.3 \%)$ & 0.14 \\
\hline $\begin{array}{ll}\text { AKI } & \text { (Creat } \\
\left.1.5^{\star} U L N\right) \$\end{array}$ & $0(0.0 \%)$ & $1(7.7 \%)$ & $0(0.0 \%)$ & $1(2.7 \%)$ & 0.39 \\
\hline $\begin{array}{l}\text { Hepatotoxicity }{ }^{\$} \\
\text { (ALT or AST } \\
1.5^{\star} \text { ULN) }\end{array}$ & $0(0.0 \%)$ & $2(15.4 \%)$ & $1(8.3 \%)$ & $3(8.1 \%)$ & 0.37 \\
\hline Glycosouria & $0(0.0 \%)$ & $1(7.7 \%)$ & $0(0.0 \%)$ & $1(2.7 \%)$ & 0.39 \\
\hline Proteinuria & $2(16.7 \%)$ & $2(15.4 \%)$ & $1(8.3 \%)$ & $5(13.5 \%)$ & 0.81 \\
\hline Haematuria & $0(0.0 \%)$ & $1(7.7 \%)$ & $1(8.3 \%)$ & $2(5.4 \%)$ & 0.60 \\
\hline Other & $2(16.7 \%)^{\#}$ & $3(23.1 \%)^{\wedge}$ & $0(0.0 \%)$ & $5(13.5 \%)$ & 0.22 \\
\hline Any Adverse Event & $9(75.0 \%)$ & $12(92.3 \%)$ & $9(75.0 \%)$ & $\begin{array}{l}30 \\
(81.1 \%)\end{array}$ & 0.44 \\
\hline
\end{tabular}

431

432

433

434

435

436

437

438

439

440

GI Gastrointestinal, AKI Acute kidney Injury

$\$$ Change in Creatinine / ALT / AST relative to baseline

${ }^{*}$ All cases of hypotension were asymptomatic and none required treatment

\# 1 Patient reported 'eyes feeling tired' and 1 patient reported pain at the IV catheter site

^ 2 Patients reported subjectively feeling cold without objective change in temperature and 1 patient developed phlebitis at the IV catheter site. 


\section{Supplementary Data}

442

443 Supplementary Figure 1: Dose adjusted drug exposure for subjects on the IDA, IDA+AZI and AZI 444 alone study arms.

445

446 Distribution of dose adjusted $\mathrm{C}_{\max }$ and AUC 0 -t of ALB-SOX (a \& b) DEC (c \& d), IVM (e \& f) and AZI (g \& f) by study ARM. The box 447 plots indicate the $25 \%$ to the $75 \%$ percentiles, and the error bars represent the $5 \%$ and $95 \%$ percentiles. Individual data points are 448 indicated by solid dots. The overall median value for both groups is indicated by the horizontal line within each box. The solid line 449 between both groups indicate grand mean value. Significance was assessed using the Kruskal-Wallis test and all $P$ values were $>$ 450 0.05. (IDA, $n=12, I D A+A Z I, n=13, A Z I, n=13)$.

451

452 
